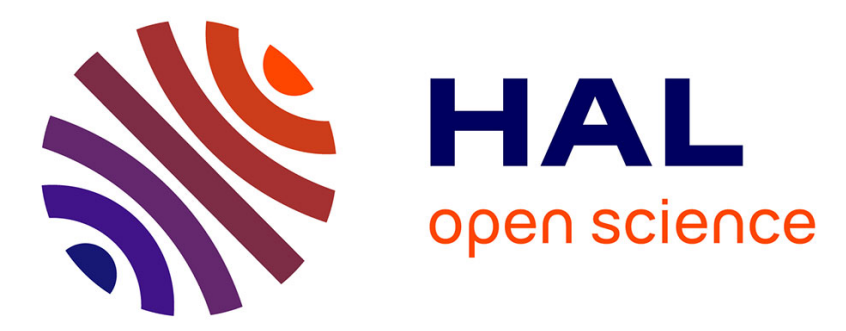

\title{
Morphological PDE and dilation/erosion semigroups on length spaces
}

Jesus Angulo

\section{To cite this version:}

Jesus Angulo. Morphological PDE and dilation/erosion semigroups on length spaces. 12th International Symposium on Mathematical Morphology, May 2015, Reykjavik, Iceland. pp.509-521, 10.1007/978-3-319-18720-4_43 . hal-01108145v3

HAL Id: hal-01108145

https://hal-mines-paristech.archives-ouvertes.fr/hal-01108145v3

Submitted on 17 Jan 2016

HAL is a multi-disciplinary open access archive for the deposit and dissemination of scientific research documents, whether they are published or not. The documents may come from teaching and research institutions in France or abroad, or from public or private research centers.
L'archive ouverte pluridisciplinaire HAL, est destinée au dépôt et à la diffusion de documents scientifiques de niveau recherche, publiés ou non, émanant des établissements d'enseignement et de recherche français ou étrangers, des laboratoires publics ou privés. 


\title{
Morphological PDE and dilation/erosion semigroups on length spaces
}

\author{
Jesús Angulo \\ MINES ParisTech, PSL-Research University, \\ CMM-Centre de Morphologie Mathématique, France \\ jesus.angulo@mines-paristech.fr
}

\begin{abstract}
This paper gives a survey of recent research on HamiltonJacobi partial differential equations (PDE) on length spaces. This theory provides the background to formulate morphological PDEs for processing data and images supported on a length space, without the need of a Riemmanian structure. We first introduce the most general pair of dilation/erosion semigroups on a length space, whose basic ingredients are the metric distance and a convex shape function. The second objective is to show under which conditions the solution of a morphological PDE in the length space framework is equal to the dilation/erosion semigroups.
\end{abstract}

Keywords: Hamilton-Jacobi PDE; Hamilton-Jacobi semigroup; length space; morphological PDE; morphological semigroup

\section{Introduction}

Let us assume a Lipschitz continuous function $f: \mathbb{R}^{n} \rightarrow \mathbb{R}$. Consider now the following initial-value Hamilton-Jacobi first-order partial differential equation (PDE)

$$
\left\{\begin{array}{l}
u_{t}(x, t) \pm H(x, D u(x, t))=0, \text { in } \mathbb{R}^{n} \times(0,+\infty), \\
u(x, 0)=f(x), \text { in } \mathbb{R}^{n},
\end{array}\right.
$$

Such family of equations usually does not admit classic (i.e., everywhere differentiable) solutions but can be studied in the framework of the theory of viscosity solutions [14]. It is well known [7,19] that if the Hamiltonian has the properties: (i) $H(x, p)=H(p)$ is convex, (ii) superlinear growth in the sense of $\lim _{|p| \rightarrow+\infty} H(p) /|p|=+\infty$, and (iii) $H(0)=0$, then the solution of Cauchy problem (1) is given for + and - respectively by the so-called Hopf-Lax-Oleinik formulas:

$$
u(x, t)=\inf _{y \in \mathbb{R}^{n}}\left[f(y)+t L\left(\frac{x-y}{t}\right)\right], u(x, t)=\sup _{y \in \mathbb{R}^{n}}\left[f(y)-t L\left(\frac{x-y}{t}\right)\right],
$$

where the Lagrangian $L(q)$ is the one-dimensional Legendre-Fenchel transform of function $H(p)$, i.e.,

$$
L(q)=H^{*}(q)=\sup _{p \in \mathbb{R}_{+}}\{p q-H(p)\}, \quad q \in \mathbb{R}_{+} .
$$


We note that, by standard results of the Legendre-Fenchel transform, $L$ is increasing, convex, superlinear and satisfies $L(0)=0$.

PDE (1) plays a central role in continuous mathematical morphology $[1,5,12,26,10]$. In particular, by taking $H(p)=1 / 2\|p\|^{2}$, such that $L(q)=1 / 2\|q\|^{2}$, a kind of canonic morphological PDE is formulated

$$
\left\{\begin{array}{l}
\frac{\partial u}{\partial t}= \pm \frac{1}{2}\|\nabla u\|^{2}, x \in \mathbb{R}^{n}, t>0 \\
u(x, 0)=f(x), \quad x \in \mathbb{R}^{n}
\end{array}\right.
$$

such that the corresponding viscosity solutions are given by

$$
\begin{array}{ll}
u(x, t)=\sup _{y \in \mathbb{R}^{n}}\left\{f(y)-\frac{\|x-y\|^{2}}{2 t}\right\} & \text { (for }+ \text { sign }), \\
u(x, t)=\inf _{y \in \mathbb{R}^{n}}\left\{f(y)+\frac{\|x-y\|^{2}}{2 t}\right\} & \text { (for }- \text { sign) },
\end{array}
$$

which just correspond to a dilation $(f \oplus b)$ and an erosion $(f \ominus b)$ of function $f(x)$ defined as

$$
\begin{aligned}
& (f \oplus b)(x)=\sup _{y \in \mathbb{R}^{n}}\{f(y)+b(y-x)\}, \\
& (f \ominus b)(x)=\inf _{y \in \mathbb{R}^{n}}\{f(y)-b(y+x)\},
\end{aligned}
$$

using as structuring function $b(x)$ the so-called multiscale quadratic (or parabolic) structuring function:

$$
p_{t}(x)=-\frac{\|x\|^{2}}{2 t} .
$$

By the way, due to its properties of semigroup, dimension separability and invariance to transform domain $[25,23,9]$, the structuring function $p_{t}(x)$ can be considered as the canonic one in morphology, playing a similar role to the Gaussian kernel in linear filtering. Other particularized forms of the Hamilton-Jacobi model (1) cover the flat morphology by disks [26]; i.e., $u_{t}= \pm\|\nabla u\|$, as well as operators with more general $P$-power concave structuring functions, i.e., $u_{t}= \pm\|\nabla u\|^{P}$. For the application of the latter model to adaptive morphology, see [15].

Morphological operators are classically defined for images supported on Euclidean spaces. We have recently introduced mathematical morphology for real valued images whose support space is a Riemannian manifold [2]. In fact, we have observed that the smoothness of the space (and its Riemannian structure) is not a fundamental requirement, since the counterpart of Euclidean quadratic operators (4) (5) are also sup/inf-convolutions where the Euclidean distance is replaced by the geodesic distance in the Riemannian manifold. Hence, dilation and erosion can be formulated for functions in a more general framework than the Euclidean or even the Riemannian case. We focus here on functions whose domain is a length (or geodesic) space and in particular we are interesting of relating the corresponding dilation/erosion with a Hamilton-Jacobi PDE formulation. 
Morphological PDE on graphs. The approximation of morphological operators using a PDE formulation has been already considered for the nonEuclidean case of weighted graphs $[28,18]$. The starting point is the definition of a gradient on the graph. Hence, the basic ingredient is an approximation of the first derivative in a vertex (or node) $u$ in the direction to a vertex $v$ as $\sqrt{w_{u v}}(f(v)-f(u))$, where $w_{u v}$ is the weight in the edge linking $u$ to $v$. Then, the gradient of a function $f$ at a vertex $u$ is defined as $\nabla f(u)=$ $\sum_{v \in N(u)} \sqrt{w_{u v}}(f(v)-f(u)), N(u)$ being the set of vertices linked to $u$. Using this gradient, a counterpart of the classical morphological PDE is formulated. The weight function in [28] is generally a distance-based kernel used for adaptive/nonlocal filters. In general, this kind of weight does not involve a natural length structure on the graph and this can be a theoretical limitation in order to link such PDE with classical Hamilton-Jacobi PDE theory. In addition, existence of viscosity solutions, and their semigroups, for those morphological PDEs on graphs were not considered in $[28,18]$.

Numerical schemes for Hamilton-Jacobi equations. There exists a large state-of-the-art on numerical schemes for Hamilton-Jacobi equations. The majority of numerical schemes which were proposed to solve Hamilton-Jacobi equations in Euclidean space are based on finite difference methods (upwind and centered discretizations, ENO or WENO schemes, etc.) The formulation of numerical discretization of Hamilton-Jacobi equations on general length spaces is out of the scope of the paper. We can nevertheless cite recent efforts on approximation schemes of Hamilton-Jacobi PDE on networks [13,22].

Hamilton-Jacobi semigroups on metric, length and geodesic spaces. During the recent years, a series of works have considered the generalization of the Hopf-Lax-Oleinik formula to a class of Hamilton-Jacobi PDEs on a length space framework. The need of these technical results was motivated by the study of geometric inequalities related to concentration measure. More precisely, connections between logarithmic Sobolev type inequalities and optimal transportentropy inequalities. See the book by Villani [29] for detailed overview on application of Hopf-Lax-Oleinik semigroup to optimal transport or papers by Ambrosio and co-workers [3,4] for the use of these semigroups in metric space calculus (heat flow, total variation, Ricci curvature bounds, etc.) on metric measure spaces. Nevertheless, up to the best of our knowledge, this theory has not been applied to practical problems in applied mathematics which use Hamilton-Jacobi PDEs, such as optimal control or mathematical morphology.

This series of works were inspired by the seminal contribution by Bobkov et al. [8] establishing the equivalence between logarithmic Sobolev inequality and hypercontractivity properties of classical Hamilton-Jacobi (semigroup) solutions. In our terminology, the semigroup used in [8] corresponds to the Euclidean erosion using a quadratic structuring function. The paper by Lott and Villani [24] is the pioneer work formulating Hamilton-Jacobi PDE acting on continuous functions on a compact measured length space and for a quadratic Hamiltonian. The approach in the same framework was extended to general con- 
vex Hamiltonians by Balogh et al. [6]. A different kind of generalization, studied more recently by Gozlan et al. [21] and Ambrosio et al. [3], involves the general case of a length space without the need of a measure structure. The particular case of the Hamilton-Jacobi semigroup on Riemannian manifolds is considered in [29]. We can mention also generalizations of Hamilton-Jacobi semigroups to specific differential geometry structures such as Heisenberg group [16]. Finally, reader is refereed to [20] for a depth insight to recent progresses on sub-solutions of Hamilton-Jacobi equations on Riemannian structures based on KAM theory.

Aim of the paper. In this context, the goal of the present paper is to give a survey on this recent theory of Hamilton-Jacobi PDEs and associated semigroups on length spaces. Therefore, we do not provide new results, except from the adjunction viewpoint, since most of the proofs can be found in the above mentioned measure theory literature. Nevertheless, in our opinion, the paper has a relevant pedagogical interest in the mathematical morphology context since this theory is useful for the generalization of morphological PDEs for images and data supported on non-Euclidean spaces, such as surfaces, graphs, point clouds, and other length spaces which can be obtained by different image embeddings [2].

\section{Preliminaries}

Metric, length and geodesic space [17]. A metric space is a set of points $X$ endowed with a distance function $d: X \times X \rightarrow[0, \infty)$. In the paper is assumed that $(X, d)$ is a complete separable metric space, locally compact (every closed ball or subset of $X$ is compact).

A length space is a metric space $(X, d)$ such that for any pair of points $x, y \in X$, we have $d(x, y)=\inf \{\operatorname{Length}(\sigma)\}$, where the infimum is taken over all rectifiable curves $\sigma:[0,1] \rightarrow X$ connecting $x$ with $y$, i.e., $\sigma(0)=x$ and $\sigma(1)=y$.

A curve $\sigma$ is called a geodesic if $\sigma$ has constant speed and if $\operatorname{Length}\left(\left.\sigma\right|_{\left[t, t^{\prime}\right]}\right)=$ $d\left(\sigma(t), \sigma\left(t^{\prime}\right)\right), \forall t, t^{\prime} \in[0,1], t \leq t^{\prime}$. A curve $\sigma$ is a geodesic if for every two points $x, y \in X$, with $\sigma(0)=x$ and $\sigma(1)=y$, one has $d\left(\sigma(t), \sigma\left(t^{\prime}\right)\right)=\left|t-t^{\prime}\right| d(x, y)$, $\forall t, t^{\prime} \in[0,1] .(X, d)$ is a geodesic space if for every pair of points $x, y \in X$ there exists a geodesic $\sigma:[0,1] \rightarrow X$ joining $x$ to $y$.

Note that every geodesic space is a length space. For the converse, we have the Hopf-Rinow Theorem: Let $X$ be a length space, complete and locally compact, then $X$ is a geodesic space.

Doubling measure space [6]. A Borel measure $\mu$ is doubling, if the measure of any open ball is positive and finite, and if there exists a constant $c_{d} \geq 1 \mathrm{such}$ that

$$
\mu(B(x, 2 r)) \leq c_{d} \mu(B(x, r))
$$

for all $x \in X$ and $r>0$. Here $B(x, r)$ denotes an open ball of radius $r$ centered in $x$. A metric measure space $(X, d, \mu)$ satisfies a doubling condition if $\mu$ is a Borel doubling measure.

Metric gradient and subgradient $[24,21,3]$. We said that $f: X \rightarrow \mathbb{R}$ is $d$ Lipschitz if there exists $C \geq 0$ satisfying $|f(x)-f(y)| \leq C d(x, y), \forall x, y \in X$. The 
least constant $C$ with this property will be denoted by $\operatorname{Lip}(f) . \operatorname{Lip}(X)$ denotes the set of real-valued Lipschitz functions on $X$.

Given $f: X \rightarrow \mathbb{R}$, we define the metric gradient of $f$ at a point $x \in X$ by

$$
|\nabla f|(x)=\limsup _{y \rightarrow x} \frac{|f(y)-f(x)|}{d(x, y)} .
$$

If $f$ is Lipschitz continuous then $|\nabla f| \in L^{\infty}(X)$.

We further introduce the metric subgradients of $f$ at $x$ defined as

$$
\left|\nabla^{-} f\right|(x)=\limsup _{y \rightarrow x} \frac{[f(y)-f(x)]_{-}}{d(x, y)}=\limsup _{y \rightarrow x} \frac{[f(x)-f(y)]_{+}}{d(x, y)},
$$

and

$$
\left|\nabla^{+} f\right|(x)=\limsup _{y \rightarrow x} \frac{[f(y)-f(x)]_{+}}{d(x, y)}=\limsup _{y \rightarrow x} \frac{[f(x)-f(y)]_{-}}{d(x, y)},
$$

where $a_{+}=\max (a, 0)$ and $a_{-}=\max (-a, 0)$. $\left|\nabla^{-} f\right|(x)$ is called descending slope and $\left|\nabla^{+} f\right|(x)$ ascending slope. Notice that $\left|\nabla^{-} f\right|(x)=\left|\nabla^{+}(-f)\right|(x)$ and $|\nabla f|(x)=\max \left\{\left|\nabla^{-} f\right|(x), \quad\left|\nabla^{+} f\right|(x)\right\}$. We can therefore work exclusively with $\left|\nabla^{-} f\right|$. Finally, we observe that if $d$ is finite, and $(X, d, \mu)$ is doubling, for any $f \in \operatorname{Lip}(X)$ then $\left|\nabla^{-} f\right|(x)=\left|\nabla^{+} f\right|(x) \mu$-almost everywhere in $X[3]$ (Proposition 2.6). Clearly, we notice that $\left|\nabla^{-} f\right|(x) \leq|\nabla f|(x)$, thus the metric subgradient is a finer notion than the gradient norm and $\left|\nabla^{-} f\right|(x)$ vanishes if $f$ has a local minimum at $x$. In a sense, $\left|\nabla^{-} f\right|(x)$ measures the downward pointing component of $f$ near $x$ : local variation of $f$ taking into account only values less than $f(x)$.

If $f$ is Lipschitz continuous then $\left|\nabla^{ \pm} f\right|(x) \leq \operatorname{Lip}(f), \forall x \in X$. Finally, when $X$ is a Riemannian manifold and $f$ is differentiable at $x$, metric subgradients $\left|\nabla^{ \pm} f\right|(x)$ are equal to the norm of the vector $\nabla f(x) \in T_{x} X$ (the tangent space at $x)[29]$.

\section{Dilation and erosion on metric spaces}

Let us consider a metric space $(X, d)$ and a given bounded function $f: X \mapsto \mathbb{R}$. We assume that $f$ is Lipschitz continuous. Let us consider a one-dimensional (shape) function $L: \mathbb{R}_{+} \rightarrow \mathbb{R}_{+}$, being increasing, superlinear, convex of class $\mathcal{C}^{1}$ such that $L(0)=0$. For all scales $t>0$, we define the dilation $D_{L ; t} f$ and the erosion $E_{L ; t} f$ operators of $f$ on $(X, d)$ according to $L$ as follows

$$
\begin{array}{ll}
D_{L ; t} f(x)=\sup _{y \in X}\left\{f(y)-t L\left(\frac{d(x, y)}{t}\right)\right\}, & \forall x \in X, \\
E_{L ; t} f(x)=\inf _{y \in X}\left\{f(y)+t L\left(\frac{d(x, y)}{t}\right)\right\}, & \forall x \in X .
\end{array}
$$

We adopt the convention $D_{L ; 0} f=E_{L ; 0} f=f$. In the context of classical mathematical morphology operators (6)- (7), correspond respectively to the multi-scale 
dilation $\left(f \oplus b_{t}\right)(x)=D_{L ; t} f(x)$ and erosion $\left(f \ominus b_{t}\right)(x)=E_{L ; t} f(x)$ of function $f$ by structuring function

$$
b_{t}(x-y)=-t L\left(\frac{d(x, y)}{t}\right) .
$$

By the way, we note that by symmetry, one has $b_{t}(x-y)=b_{t}(y-x)$. A typical example of a shape function is $L(q)=q^{P} / P, P>1$, such that

$$
b_{t}(x-y)=-\frac{d(x, y)^{P}}{P t^{P-1}} .
$$

The canonic shape function corresponds to the case $P=2: b_{t}(x-y)=-\frac{d(x, y)^{2}}{2 t}$.

Properties. The following properties hold for any metric space $(X, d)$.

1. (Adjunction) For any two real-valued functions $f$ and $g$ on $(X, d)$, the pair $\left(E_{L ; t}, D_{L ; t}\right)$ forms an adjunction, i.e.,

$$
D_{L ; t} f(x) \leq g(x) \Leftrightarrow f(x) \leq E_{L ; t} g(x), \quad \forall x \in X .
$$

2. (Duality by involution) For any function $f$ and $\forall x \in X$, one has

$$
D_{L ; t} f(x)=-E_{L ; t}(-f)(x) ; \text { and } E_{L ; t} f(x)=-D_{L ; t}(-f)(x), \forall t>0 .
$$

3. (Increaseness) If $f(x) \leq g(x), \forall x \in X$, then

$$
D_{L ; t} f(x) \leq D_{L ; t} g(x) ; \text { and } E_{L ; t} f(x) \leq E_{L ; t} g(x), \forall x \in X, \forall t>0 .
$$

4. (Extensivity and anti-extensivity)

$$
D_{L ; t} f(x) \geq f(x) ; \text { and } E_{L ; t} f(x) \leq f(x), \forall x \in X, \forall t>0 .
$$

5. (Ordering property) If $0<s<t$ then $\forall x \in X$

$$
\inf _{X} f \leq E_{L ; t} f(x) \leq E_{L ; s} f(x) \leq f(x) \leq D_{L ; s} f(x) \leq D_{L ; t} f(x) \leq \sup _{X} f .
$$

6. (Convergence) For any function $f$ and $\forall x \in X, D_{L ; t} f(x)$ and $E_{L ; t} f(x)$ converge monotonically to $f(x)$ as $t \rightarrow 0$. In particular $\lim _{t \rightarrow 0} D_{L ; t} f=f$ and $\lim _{t \rightarrow 0} E_{L ; t} f=f$.

7. (Lipschitz) The maps $(x, t) \mapsto D_{L ; t} f(x)$ and $(x, t) \mapsto E_{L ; t} f(x)$ are in $\operatorname{Lip}\left(X \times \mathbb{R}_{+}\right)$.

8. (Semigroup) For any function $f$ and $\forall x \in X$, and for all pair of scales $s, t>0$, - If $X$ is metric space:

$$
D_{L ; t} D_{L ; s} f \leq D_{L ; t+s} f ; \text { and } E_{L ; t} E_{L ; s} f \geq E_{L ; t+s} f .
$$

- If $X$ is a length space:

$$
D_{L ; t} D_{L ; s} f=D_{L ; t+s} f ; \text { and } E_{L ; t} E_{L ; s} f=E_{L ; t+s} f .
$$


Proof. For property 1, on adjunction, we have that the inequality $D_{L ; t} f(x) \leq g(x)$ means that

$$
\sup _{y \in X}\left\{f(y)-t L\left(\frac{d(x, y)}{t}\right)\right\} \leq g(x), \quad \forall x \in X,
$$

It involves that $f(y)-t L(d(x, y) / t) \leq g(x)$ for every $x, y \in X$. This is equivalent to rewrite $f(y) \leq g(x)+t L(d(x, y) / t)$. Therefore, after substitution of $z=x$, we finally have

$$
f(y) \leq \inf _{z \in X}\left\{g(y)+t L\left(\frac{d(z, y)}{t}\right)\right\}=E_{L ; t} g(y) .
$$

For the duality of 2, we have that $D_{L ; t}(-f)(x)$ is equal to

$$
\sup _{y \in X}\left\{-f(y)-t L\left(\frac{d(x, y)}{t}\right)\right\}=-\inf _{y \in X}\left\{f(y)+t L\left(\frac{d(x, y)}{t}\right)\right\}=-E_{L ; t}(f)(x) .
$$

The properties 3 and 4 of increaseness and extensivity/anti-extensivity are obvious from the properties of supremum/infimum.

The proof of ordering property 5 is based on the following semigroup property [6](Theorem 2.5.(ii)): For $0 \leq s<t$

$$
E_{L ; t} f(x)=\min _{y \in X}\left[E_{L ; s} f(y)+(t-s) L\left(\frac{d(x, y)}{t-s}\right)\right] .
$$

Now for a fixed $z \in X$, we have

$$
E_{L ; t} f(z)=\min _{\zeta \in X}\left[E_{L ; s} f(\zeta)+(t-s) L\left(\frac{d(\zeta, z)}{t-s}\right)\right] \leq(t-s) L(0)+E_{L ; s} f(\zeta) .
$$

By choosing $z=\zeta$ and using $L(0)=0$, we have $E_{L ; t} f(z) \leq E_{L ; s} f(z)$.

In order to prove the semigroup property 8 , following [24], we consider for the sake of simplicity the case of the canonic shape function $L(q)=q^{2} / 2$. Now, triangle inequality implies that for all $x, y \in X$ and $s, t>0$,

$$
\frac{d(x, y)^{2}}{2(t+s)} \leq \inf _{z \in X}\left[\frac{d(x, z)^{2}}{2 t}+\frac{d(z, y)^{2}}{2 s}\right] .
$$

The equality in (14) in length spaces comes from choosing a minimal geodesic between $x$ and $y$, and a point $z$ on this geodesic with $d(x, z)=\frac{t}{s+t} d(x, y)$. Finally, from (14), we obtain

$$
\begin{aligned}
E_{L ; t+s} f(x) & =\inf _{y \in X}\left[f(y)+\frac{d(x, y)^{2}}{2(t+s)}\right]=\inf _{y \in X} \inf _{z \in X}\left[f(y)+\frac{d(x, z)^{2}}{2 t}+\frac{d(z, y)^{2}}{2 s}\right] \\
& =E_{L ; t} E_{L ; s} f(x) .
\end{aligned}
$$

For the a general function $L$, see [6].

The proof of properties 6 and 7 on convergence and Lipschitz are not included by the limited length of the paper, see [24,6].

Bibliographic remark. Following [8] and [24], our metric erosion (13) corresponds to the semigroup $Q_{t} f$, which is the basic ingredient in the theory of geometric inequalities related to concentration measure. The dual and adjoint semigroup (our dilation (12)) is only considered in [21] and is denoted by $P_{t} f$. $Q_{t} f$ is named as Hamilton-Jacobi semigroup on length spaces in [24] whereas other works $[6,21,3]$ use the most classical terminology from max-plus mathematics on Hilbert spaces: $Q_{t} f$ is the Hopf-Lax-Oleinik semigroup on length (or geodesic) spaces. 


\section{Morphological PDE on metric spaces}

We introduce the morphological PDE on a metric space $(X, d)$ as the the following initial-value Hamilton-Jacobi first-order equation:

$$
\left\{\begin{array}{l}
\frac{\partial}{\partial t} u(x, t) \pm H\left(\left|\nabla^{-} u(x, t)\right|\right)=0, \text { in } X \times(0,+\infty), \\
u(x, 0)=f(x), \text { in } X,
\end{array}\right.
$$

where the initial condition $f: X \rightarrow \mathbb{R}$ is a continuous bounded function and $H: \mathbb{R}_{+} \rightarrow \mathbb{R}_{+}$is the Legendre transform of function $L(q)$ :

$$
H(p)=\max _{q \in \mathbb{R}_{+}}\{p q-L(q)\}, p \in \mathbb{R}_{+} .
$$

Our objective now is to show under which conditions the solution of a Hamilton-Jacobi PDE in the metric space framework is equal to the dilation and erosion semigroups. We first consider the results from [24] and [6].

Theorem 1 (Lott and Villani, 2007; Balogh et al.,2012). The solutions of PDE problem (15) are the dilation (12) and erosion (13) semigroups:

$$
\begin{aligned}
& u(x, t)=D_{L ; t} f(x) \quad(\text { for }- \text { sign }), \\
& u(x, t)=E_{L ; t} f(x) \quad(\text { for }+ \text { sign }),
\end{aligned}
$$

in the following cases.

1. If $(X, d)$ is a length space: solutions hold for all $x \in X$ and for almost everywhere $t>0$.

2. If $(X, d, \mu)$ satisfies a doubling condition and supports a local Poincaré inequality: solutions hold for $\mu$-almost everywhere $x \in X$ and for all $t>0$.

Proof. For the sake of pedagogy, let us recall the proof of the solution as an erosion $u(x, t)=E_{L ; t} f(x)$ in the case 1 . The corresponding one for case 2 can be found in [24] and [6], where the role of doubling measure and Poincaré inequality are explained.

We first show that the inequality

$$
\frac{\partial}{\partial t} u(x, t)+H\left(\left|\nabla^{-} u\right|(x, t)\right) \leq 0
$$

holds for every $x \in X$ and a.e. $t \in \mathbb{R}_{+}$for $u(x, t)=E_{L ; t} f(x)$.

Fix $x \in X$ and let $t \in \mathbb{R}_{+}$be a point of differentiability of $u(x, \cdot)$. If $\left|\nabla^{-} u\right|(x, t)=0$, (18) reduces to $u_{t}(x, t) \leq 0$ since $H(0)=0$. This clearly holds since $u(x, \cdot)$ is nonincreasing. We can thus assume that $\left|\nabla^{-} u\right|(x, t)>0$, and there exists a sequence $x_{n} \rightarrow x$ for which $u\left(x_{n}, t\right)<u(x, t)$ and $\left|\nabla^{-} u\right|(x, t)=\lim _{n \rightarrow \infty} \frac{u(x, t)-u\left(x_{n}, t\right)}{d\left(x_{n}, x\right)}$. For the moment, consider any positive sequence $\left(h_{n}\right)$ with $h_{n} \rightarrow 0$. By the semigroup property [6](Theorem 2.5.(ii)): For $0 \leq s<t$

$$
E_{L ; t} f(x)=\min _{y \in X}\left[E_{L ; s} f(y)+(t-s) L\left(\frac{d(x, y)}{t-s}\right)\right] .
$$


we get

$$
u\left(x, t+h_{n}\right)=\min _{y \in X}\left\{h_{n} L\left(\frac{d(x, y)}{h_{n}}\right)+u(y, t)\right\} \leq h_{n} L\left(\frac{d\left(x, x_{n}\right)}{h_{n}}\right)+u\left(x_{n}, t\right),
$$

which implies that

$$
\frac{u\left(x, t+h_{n}\right)-u(x, t)}{h_{n}} \leq-\left[\frac{u(x, t)-u\left(x_{n}, t\right)}{h_{n}}-L\left(\frac{d\left(x, x_{n}\right)}{h_{n}}\right)\right] .
$$

Since $H(p)=\max _{q \in \mathbb{R}_{+}}\{p q-L(q)\}, \forall p \in \mathbb{R}_{+}$, for each $n$ it is possible to choose $h_{n}>0$ such that

$$
H\left(\frac{u(x, t)-u\left(x_{n}, t\right)}{d\left(x_{n}, x\right)}\right)=\frac{u(x, t)-u\left(x_{n}, t\right)}{h_{n}}-L\left(\frac{d\left(x, x_{n}\right)}{h_{n}}\right)
$$

holds. Furthermore, it is easy to see directly from (21) that $x_{n} \rightarrow x$ implies $h_{n} \rightarrow 0$. Finally, combining (20) and (21) we obtain

$$
\frac{u\left(x, t+h_{n}\right)-u(x, t)}{h_{n}}+H\left(\frac{u(x, t)-u\left(x_{n}, t\right)}{d\left(x_{n}, x\right)}\right) \leq 0 .
$$

As $x_{n} \rightarrow x$ and $h_{n} \rightarrow 0$, letting $n \rightarrow \infty$ gives us (18).

The converse inequality to (18) can be written as

$$
\liminf _{s \rightarrow 0^{+}} \frac{E_{L ; t+s} f(x)-E_{L ; t} f(x)}{s} \geq-H\left(\left|\nabla^{-} E_{L ; t} f\right|(x)\right) .
$$

Let us fix $x \in X$ and $t \in \mathbb{R}_{+}$. Since $(x, t) \mapsto E_{L ; t} f(x)$ is a Lipschitz function, the limit inferior in (22) is finite and we can choose a positive sequence $\left(h_{n}\right)$ such that $h_{n} \rightarrow 0$ and

$$
\liminf _{s \rightarrow 0^{+}} \frac{E_{L ; t+s} f(x)-E_{L ; t} f(x)}{s}=\lim _{n \rightarrow \infty} \frac{E_{L ; t+h_{n}} f(x)-E_{L ; t} f(x)}{h_{n}} .
$$

Next, applying again the semigroup property (19) we can write

$$
E_{L ; t+h_{n}} f(x)=\min _{y \in X}\left\{h_{n} L\left(\frac{d(x, y)}{h_{n}}\right)+E_{L ; t} f(y)\right\} .
$$

For each $n$ we choose a point $y_{n} \in X$ for which the minimum is attained. The superlinearity of $L$ implies that $y_{n} \rightarrow x$. As $E_{L ; t} f(x)$ is decreasing in $t$, we have $E_{L ; t+h_{n}} f(x) \leq E_{L ; t} f(x)$, and hence

$$
E_{L ; t} f\left(y_{n}\right) \leq h_{n} L\left(\frac{d(x, y)}{h_{n}}\right)+E_{L ; t} f\left(y_{n}\right) \leq E_{L ; t} f(x) .
$$

Since $H(p)=\max _{q \in \mathbb{R}_{+}}\{p q-L(q)\}$, we have $H(p)+L(q) \geq p q, \forall p, q \in \mathbb{R}_{+}$. Together with (25) this implies that

$$
H\left(\frac{E_{L ; t} f(x)-E_{L ; t} f\left(y_{n}\right)}{d\left(x, y_{n}\right)}\right)+L\left(\frac{d\left(x, y_{n}\right)}{h_{n}}\right) \geq \frac{E_{L ; t} f(x)-E_{L ; t} f\left(y_{n}\right)}{h_{n}},
$$

and we have

$$
L\left(\frac{d\left(x, y_{n}\right)}{h_{n}}\right)+\frac{E_{L ; t} f\left(y_{n}\right)-E_{L ; t} f(x)}{h_{n}} \geq-H\left(\frac{\left[E_{L ; t} f(x)-E_{L ; t} f\left(y_{n}\right)\right]_{+}}{d\left(x, y_{n}\right)}\right) .
$$


Together with (24) this implies

$$
\begin{aligned}
\frac{E_{L ; t+h_{n}} f(x)-E_{L ; t} f(x)}{h_{n}} & =\frac{1}{h_{n}}\left(h_{n} L\left(\frac{d\left(x, y_{n}\right)}{h_{n}}\right)+E_{L ; t} f\left(y_{n}\right)-E_{L ; t} f(x)\right) \\
& \geq-H\left(\frac{\left[E_{L ; t} f(x)-E_{L ; t} f\left(y_{n}\right)\right]_{+}}{d\left(x, y_{n}\right)}\right) .
\end{aligned}
$$

Letting now $n \rightarrow \infty$ and using (23) we obtain

$$
\begin{aligned}
\liminf _{s \rightarrow 0^{+}} \frac{E_{L ; t+s} f(x)-E_{L ; t} f(x)}{s} & \geq \limsup _{n \rightarrow \infty}\left(-H\left(\frac{\left[E_{L ; t} f(x)-E_{L ; t} f\left(y_{n}\right)\right]_{+}}{d\left(x, y_{n}\right)}\right)\right) \\
& \geq-H\left(\left|\nabla^{-} E_{L ; t} f\right|(x)\right) .
\end{aligned}
$$

Notice that, if $u(x, t)=E_{L ; t} f(x)$, and $t$ is a point of differentiability of $t \rightarrow u(x, t)$ for a fixed $x$, then it follows that $\frac{\partial}{\partial t} u(x, t)+H\left(\left|\nabla^{-} u\right|(x, t)\right) \geq 0$. Since $u$ is Lipschitzcontinuous, the above inequality holds for all $x \in X$ and a.e. $t \in \mathbb{R}_{+}$.

Combining inequalities (18) and (22), we obtain the equality.

Theorem 1 tell us that the solutions of the morphological PDE are the dilation and erosion for all $x \in X, X$ being a length space, and for all $t$ outside a set $N_{t}$ of measure 0 . In fact, it has been proven more recently [21] that the result holds without the need of measure theory.

Theorem 2 (Gozlan et al.,2014). In a geodesic space $(X, d)$, the solutions (16)(17) hold for all $x \in X$ and for all $t>0$.

Finally, in analogy to the Euclidean case, the canonic morphological PDE in a length space $(X, d)$ is given by

$$
\begin{cases}\frac{\partial}{\partial t} u(x, t)= \pm \frac{1}{2}\left|\nabla^{-} u(x, t)\right|^{2}, & x \in X, t>0 \\ u(x, 0)=f(x), & x \in X,\end{cases}
$$

such that the corresponding semigroup solutions are given by

$$
\begin{array}{ll}
u(x, t)=\sup _{y \in X}\left\{f(y)-\frac{d(x, y)^{2}}{2 t}\right\} & (\text { for }+ \text { sign }), \\
u(x, t)=\inf _{y \in X}\left\{f(y)+\frac{d(x, y)^{2}}{2 t}\right\} & (\text { for }- \text { sign }) .
\end{array}
$$

Bibliographic remark. We note that the case of real-valued extended functions $f: X \rightarrow \overline{\mathbb{R}}, \overline{\mathbb{R}}=$, with $\mathbb{R} \cup\{+\infty,-\infty\}$, requires a more technical treatment, see Section 3 in Ambrosio et al. [3].

\section{Conclusions and perspectives}

We have introduced the most general pair of dilation/erosion operators on a metric space, whose basic ingredients are the metric distance and a convex shape 
function. We have stated that the families of scale-space dilations $\left\{D_{L ; t}\right\}_{t>0}$ and erosions $\left\{E_{L ; t}\right\}_{t>0}$ are semigroups acting on bounded functions only for length spaces. We have introduced the morphological PDE on length spaces and reviewed the theoretical results which provide us a complete transposition from the Euclidean to the geodesic counterpart, linking the morphological PDE to its viscous solutions as dilation/erosion semigroups.

The theory of this paper can be used in many practical situations under the assumption of working on a geodesic space, but without the need of any smoothness of the space or curvature constraints. Discretization and numerical schemas for the morphological PDE on useful cases such as graphs and meshes will be considered in future work. The starting point can be the recent approximation schemes of Hamilton-Jacobi PDE on networks [13,22].

From a theoretical viewpoint, we plan in our perspectives to explore three different lines. First, Eikonal equation is another Hamilton-Jacobi PDE which is the basic ingredient for morphological segmentation (computation of a weighted distance function and watershed segmentation [27]), the corresponding PDE on length spaces is therefore important for us. Second, we will focuss on the particular case of metric spaces of non-positive curvature and $C A T(0)$ spaces [11]. That includes combinatorial spaces such as trees and simplicial complexes. Third, we will study the counterpart of the theory for bounded functions on ultrametric spaces, which are also relevant to mathematical morphology (dendograms and hierarchies).

\section{References}

1. L. Alvarez, F. Guichard, P-L. Lions, J-M. Morel. Axioms and fundamental equations of image processing. Arch. for Rational Mechanics, 123(3): 199-257, 1993.

2. J. Angulo, S. Velasco-Forero. Riemannian Mathematical Morphology. Pattern Recognition Letters, 47:93-101, 2014.

3. L. Ambrosio, N. Gigli, G. Savaré. Calculus and heat flow on metric measure spaces and applications to spaces with Ricci curvature bounded below. Inventiones mathematica, 195 (2):289-391, 2014.

4. L. Ambrosio, S. Di Marino. Equivalent definitions of BV space and total variation in metric measure spaces. Journal of Functional Analysis, 266(7):4150-4188, 2014.

5. A.B. Arehart, L. Vincent, B.B. Kimia. Mathematical morphology: The HamiltonJacobi connection. In Proc. of IEEE 4th Inter. Conf. on Computer Vision (ICCV'93), 215-219, 1993.

6. Z.M. Balogh, A. Engulatov, L. Hunziker, O.E. Maasalo. Functional Inequalities and Hamilton-Jacobi Equations in Geodesic Spaces. Potential Analysis, 36(2): 317-337, 2012.

7. M. Bardi, L.C. Evans. On Hopf's formulas for solutions of Hamilton- Jacobi equations. Nonlinear Analysis, Theory, Methods and Applications, 8(11):1373-1381, 1984.

8. S.G. Bobkov, I.Gentil, M.Ledoux. Hypercontractivity of Hamilton-Jacobi equations. J. Math. Pures Appl., 80(7):669-696, 2001.

9. R. van den Boomgaard, L. Dorst. The morphological equivalent of Gaussian scalespace. In Proc. of Gaussian Scale-Space Theory, 203-220, Kluwer, 1997. 
10. M. Breuß, J. Weickert. Highly accurate PDE-based morphology for general structuring elements. In Proc. of Scale Space and Variational Methods in Computer Vision, LNCS Vol. 5567, 758-769, Springer, Berlin, 2009.

11. M.R. Bridson, André Haefliger. Metric spaces of non-positive curvature. Grundlehren der mathematischen Wissenschaften, Vol.319, Springer-Verlag, 1999.

12. R.W. Brockett, P. Maragos. Evolution equations for continuous-scale morphology. IEEE Trans. on Signal Processing, 42(12): 3377-3386, 1994.

13. F. Camillia, A. Festab, D. Schiebornc. An approximation scheme for a HamiltonJacobi equation defined on a network. Applied Numerical Mathematics, 73: 33-47, 2013 ,

14. M.G. Crandall, H. Ishii, P.-L. Lions. User's guide to viscosity solutions of second order partial differential equations. Bulletin of the American Mathematical Society, 27(1):1-67, 1992.

15. E.H.S. Diop, J. Angulo. Multiscale Image Analysis Based on Robust and Adaptive Morphological Scale-Spaces. HAL preprint, hal-00975728, 2014.

16. F. Dragoni. Metric Hopf-Lax formula with semicontinuous data. Discrete Contin. Dyn. Syst., 17(4):713-729, 2007.

17. D. Burago, Y. Burago, S. Ivanov. A course in metric geometry, Graduate Studies in Mathematics 33, AMS, Providence, 2001.

18. A. Elmoataz, X. Desquesnes, O. Lézoray, O. Non-Local Morphological PDEs and Laplacian Equation on Graphs With Applications in Image Processing and Machine Learning. IEEE Journal of Selected Topics in Signal Processing, 6(7):764$779,2012$.

19. L.C. Evans. Partial differential equations. Vol. 19 of Graduate Studies in Mathematics, American Mathematical Society, Providence, RI, 1998.

20. A. Fathi. Weak KAM Theorem in Lagrangian Dynamics. Series: Cambridge Studies in Advanced Mathematics. Cambridge University Press, 2014.

21. N. Gozlan, C. Roberto, P.-M. Samson. Hamilton-Jacobi equations on metric spaces and transport-entropy inequalities. Revista Matematica Iberoamericana, 30(1):133$163,2014$.

22. M. Herty, U. Ziegler, S. Göttlich. Numerical discretization of Hamilton-Jacobi equations on networks. Networks and Heterogeneous Media, 8(3):685-705, 2013.

23. P.T. Jackway, M. Deriche. Scale-Space Properties of the Multiscale Morphological Dilation-Erosion. IEEE Trans. Pattern Anal. Mach. Intell., 18(1): 38-51, 1996.

24. J. Lott, C. Villani. Hamilton-Jacobi semigroup on length spaces and applications. J. Math. Pures Appl., 88(3):219-229, 2007.

25. P. Maragos. Slope Transforms: Theory and Application to Nonlinear Signal Processing. IEEE Trans. on Signal Processing, 43(4): 864-877, 1995.

26. P. Maragos. Differential morphology and image processing. IEEE Trans. on Image Processing, 5(1): 922-937, 1996.

27. F. Meyer, P. Maragos. Multiscale Morphological Segmentations Based on Watershed, Flooding, and Eikonal PDE. In Proc. of Scale-Space'99, LNCS 1682, 351-362, Springer 1999.

28. V.-T. Ta, A. Elmoataz, O. Lezoray. Nonlocal PDEs-Based Morphology on Weighted Graphs for Image and Data Processing. IEEE Trans. on Image Processing, 20(6): 1504-1516, 2011.

29. C. Villani. Optimal transport. Old and new. Vol. 338 of Grundlehren der Mathematischen Wissenschaften [Fundamental Principles of Mathematical Sciences]. Springer-Verlag, Berlin, 2009. 